\title{
New insights into the Pt(hkl)-alkaline solution interphases from the laser induced temperature jump method.
}

Francisco J. Sarabia, Paula Sebastián ${ }^{1}$, Víctor Climent*, and Juan M. Feliu*

Instituto Universitario de Electroquímica, Universidad de Alicante, Carretera San Vicente del Raspeig s/n, E-03690 San Vicente del Raspeig, Alicante, Spain

1 Present address: Department of Chemistry, University of Copenhagen, Universitetsparken 5, 2100, Copenhagen

\section{Abstract}

The interfacial properties of platinum single crystal electrodes in contact with alkaline aqueous solutions $(\mathrm{pH}=13)$ have been investigated using the laser induced temperature jump method. This technique offers insights into the net orientation of water dipoles in contact with the electrode surface by recording the coulostatic potential changes after a sudden increase of the interfacial temperature in the submicrosecond time scale. This information is intimately related with the magnitude and sign of charge separation at the interphase and the resulting electric field. In all cases, water shows a net orientation with the hydrogen towards the metal at the lowest investigated potential value, reflected in negative potential transients. The magnitude of the water orientation decreases as the applied potential increases. Eventually, the sign of the potential transient changes, reflecting a reorientation of the water dipoles. The potential where such inversion takes place follows the order $\operatorname{Pt}(110)<\operatorname{Pt}(100)<\operatorname{Pt}(111)$ in accordance with the observed behavior in acid solution and the trend of the work function. For $\operatorname{Pt}(111)$ the change of sign of the laser induced potential transient takes place at the onset of hydroxyl adsorption. For the three surfaces, when the $\mathrm{pH}$ is decreased to ca. $\mathrm{pH}=11$, a slow response is detected at potentials values above the inversion point. This could be due to a fast adsorption process or to a slow reorientation of water. After the introduction of steps on the (111) terrace, the inversion shifts to the double layer region, allowing the unambiguous identification of the inversion with a change on the net orientation of the water molecules. For stepped surfaces, a second inversion of the laser induced potential transient is observed that could be related with an effect of the local charge on steps 
disrupting the ordering of the water network. Comparison with analogous results in acid solution gives information about the local distribution of charges on the stepped surfaces.

Keywords: Platinum single crystal electrodes; potential of zero charge; potential of maximum entropy; alkaline solutions; polarization of interfacial water; stepped surfaces.

Corresponding authors: victor.climent@ua.es; juan.feliu@ua.es

\section{Introduction}

Charge separation is probably the most important feature associated to the electrochemical interphase, inducing a strong electric field that affects in great extent the solvent molecules near the electrode surface. In contact with aqueous electrolytes, the surface of the electrode is covered with a layer of water dipoles polarized by the electric field [1,2]. Such a network of water molecules highly influences the reactivity of the electrode, strongly affecting the transport of reactants and products across the electrified interphase. For this reason, the detailed description of the relationships between the different parameters that affect the charge separation, such as electrode potential, solution composition and $\mathrm{pH}$, all of them influenced by the crystallographic structure of the electrode, is necessary to understand the electrocatalytic properties of different electrode materials.

The relationship between charge and potential is determined by two parameters: the potential of zero charge (pzc) [3,4] and the differential capacity of the interphase. In recent decades, significant efforts have been dedicated to understand the relationships between those parameters and the surface structure of platinum electrodes [5-12], since this metal is of great importance for a large number of reactions of technological interest. Early studies in our laboratory showed that charge displacement using carbon monoxide allows to determine the total charge on a platinum electrode surface as a function of the applied electrode potential [5,6,9,13-16]. However, this technique cannot differentiate between the true (so called free) charge separation at the interphase, from the charge stored in covalent bonds with adsorbed species (faradic charge). While the former is of great importance to determine the surface electric field, only the total charge (free plus adsorption charge) is determined with the displacement experiment. 
The only exception where it has been possible to separate total and free charge using the CO charge displacement information is the case of $\mathrm{Pt}(111)$ in electrolytic solutions that do not contain specifically adsorbing anions $[9,15,16]$. In this case, three regions can be clearly distinguished in the voltammogram: the hydrogen adsorption region at low potentials, the hydroxyl adsorption region at higher potentials and a double layer region in between. In the double layer region, it is reasonable to assume that there are no adsorption processes and, therefore, the charge in this region can be considered as purely free charge. Under reasonable assumptions, values of free charge in the double layer region can be extrapolated into the hydrogen or the hydroxyl regions to determine the potential of zero free charge (pzfc) $[9,15,16]$. For this reason, this particular interphase has been comparatively the most investigated and the effect of $\mathrm{pH}$ has been extensively studied to understand the relation between charge and reactivity.

Additional indirect measurements have also been proposed to address the determination of charges in the case of platinum electrodes. On one side, the sensitivity of some reactions to the electrode charge has been exploited to gain further information about the interphase. $\mathrm{N}_{2} \mathrm{O}$ [17-19] and peroxodisulfate reductions [10,20-23] have been successfully used with this purpose. In electrocatalysis, $\mathrm{H}_{2} \mathrm{O}_{2}$ and $\mathrm{O}_{2}$ [24] reduction have been also shown to be sensitive to the interfacial charge. On the other hand, the temperature jump (T-jump) experiments provide valuable information on the surface electric field. In this technique, the interfacial temperature is suddenly risen by using pulsed laser illumination. Then, the coulostatic potential change (the change of the open circuit potential) in response to the T- jump contains information about the orientation of water dipoles at the interface [8,25-30]. The main advantage of this methodology is that, by reducing the time scale of the experiment to the submicrosecond range, using a suitable pulsed laser, adsorption processes can be decoupled from purely capacitive responses and, in most of the situations, a response sensitive to the free charge can be obtained. However, this charge on the electrode can be affected and /or screened by specifically adsorbed species, such as anion adsorption, or by surface oxidation [31].

The consistency between charge displacement and the results of the temperature jump method has been tested in acid solutions and at $\mathrm{pH}$ closer to neutrality $[8,27]$. However, alkaline solutions have been less investigated, despite its importance for the development of alkaline fuel cells and electrolyzers. The extrapolation of free charge measured in the double layer with the CO displacement method explained above 
suggests the independence of the pzfc with the $\mathrm{pH}$ [15]. In mildly acidic solution, the pzfc lies in the double layer region [14]. However, since $\mathrm{OH}$ adsorption and surface oxidation processes shift with $\mathrm{pH}$ according to Nernst law, and assuming that the pzfc remains constant, the extrapolated value of the pzfc in highly alkaline solution would appear located in the potential region where the surface is covered with oxygencontaining species. It has recently been proposed that the oxide layer on the surface affects the distribution of charges at the interphase since the oxide formation induces negative charges on the metal side, even at potential values above the pzfc [31]. Therefore, the value of the pzfc extrapolated from the CO displacement technique would correspond to an unrealistic situation in which the surface remains uncovered by oxide at high applied potentials.

We provide here, for the first time, a comprehensive study of the interphase of $\operatorname{Pt}(111)$ with alkaline solutions using the temperature jump experiment. The effect of $\mathrm{pH}$ and surface structure (introduction of steps) is also investigated.

\section{Experimental Section}

Working electrodes were prepared from small beads, ca. $2 \mathrm{~mm}$ in diameter, obtained by the method described by Clavilier et al. [32,33] Before each experiment, the working electrode was annealed in a Bunsen flame (propane-air), cooled down in a hydrogen/argon (1:3) atmosphere [33,34] and transferred to the cell, protected by a drop of ultra-pure water saturated with these gases.

All experiments were performed in a three-electrode electrochemical cell, with the working electrode under meniscus configuration. A hydrogen-charged Pd wire was used as reference electrode [35] whereas a Pt wire was used as a counter electrode. For the laser induced temperature jump experiment, a second Pt wire was polarized as a second working electrode and, then, used as reference for the measurement of the potential transient, as described below. All these electrodes were decontaminated by flame annealing before introducing them into the cell. The Pd reference wire was then charged with $\mathrm{H}_{2}$ by galvanostatic polarization using a current of $-10 \mathrm{~mA}$ during 15 minutes in the same working solution. In all cases, potentials where converted either to RHE or SHE scales, as indicated in the different figures.

Cyclic voltammograms were recorded using a $\mu$-Autolab III potentiostat (Eco-Chemie, Utrecht, The Netherlands) under the current integration mode. In this mode, the 
discreteness of the potential steps during the staircase potential modulation are filtered and essentially the same voltammogram as with a pure linear scan is obtained. Solutions were prepared using sodium hydroxide monohydrated (Merck suprapur) and from concentrated perchloric acid (Merck suprapur) diluted in ultrapure water $(18.2 \mathrm{M} \Omega \mathrm{cm}$ ) obtained from Elga Purelab Ultra Analytic system.

To record the laser transients the procedure used was as follows [27]. Before recording the laser transient, the cyclic voltammogram was recorded to ensure the cleanliness and stability of the system. At the beginning, both working electrodes are polarized at a given potential and $200 \mu$ sefore the Q-switch of the laser, both electrodes are disconnected from the potentiostat using solid state relays. The laser only reaches the Pt(111) electrode surface while the second working platinum wire is unaffected by the laser. The potential difference between both working electrodes is measured at open circuit potential as a consequence of the temperature jump originated by the laser firing. The measurement is repeated with the natural frequency of the laser, which fires at $10 \mathrm{~Hz}$. This frequency is low enough to ensure that the temperature relaxes to the initial value between transients. The potentiostat is reconnected between successive laser pulses to keep the potential at the desired value. In that way, between 128 and 256 potential transients are recorded and averaged using a Tektronix Model TDS 3054B oscilloscope. Finally, after recording all desired potential transients, the cyclic voltammetry was again registered aiming to check the surface stability during the experiment.

The duration of the pulse is $5 \mathrm{~ns}$ and the laser used is a $532 \mathrm{~nm}$ frequency Nd-YAG (Brilliant B from Quantel). A special arrangement of lenses is used both to reduce the initial diameter of the laser beam from $6 \mathrm{~mm}$ to $4 \mathrm{~mm}$ and to direct the laser beam to the cell. The electrochemical cell is inside a faraday box in order to reduce the noise in the measurements. The energy density of the laser beam is reduced approximately to $15-20$ $\mathrm{mJ} / \mathrm{cm}^{2}$ and $7-8 \mathrm{~mJ} / \mathrm{cm}^{2}$ for the basal planes and stepped surfaces, respectively, by combining the effect of an attenuator from Newport Corporation (Model M-935-10) and the regulation of the Q-switch time. The laser energy was measured with a pyroelectric sensor head (Model LM-P10i).

\section{Results and Discussion}


$\operatorname{Pt}(111)$

Figure 1 shows the cyclic voltammograms obtained for a Pt(111) surface in contact with aqueous solutions at $\mathrm{pH}$ values between 11 and 13. To prepare the solutions with $\mathrm{pH}$ below 13, the $0.1 \mathrm{M} \mathrm{NaOH}$ solution was partially neutralized with suitable amounts of $\mathrm{HClO}_{4}$ aiming to avoid solution contamination and the introduction of specifically adsorbing anions. The characteristic voltammograms reveal that the surface is free of contaminants and well-oriented [15,36,37]. Three regions can be distinguished in the voltammogram. Taking as reference the $0.1 \mathrm{M} \mathrm{NaOH}$ solution, those regions are: i) below $-0.4 \mathrm{~V}$, currents correspond to the hydrogen adsorption / desorption region; ii) between -0.4 and $-0.2 \mathrm{~V}$, the double layer region signals the absence of adsorption processes and iii) above $-0.2 \mathrm{~V} \mathrm{OH}$ adsorption / desorption region dominates the voltammogram. The main voltammetric features shift ca. $60 \mathrm{mV}$ per $\mathrm{pH}$ unit in the SHE scale, following a Nernstian behavior. The small peak at ca. $-0.4 \mathrm{~V}$ (in $0.1 \mathrm{M} \mathrm{NaOH}$ ) corresponds to the response of a small amount of (100) defects on the surface, while the peak at $-0.53 \mathrm{~V}$ corresponds to $(110)$ defects $[36,38]$. The small relative height of these peaks confirms the good quality of the surface. Results below $\mathrm{pH} 11$ are not shown because local $\mathrm{pH}$ changes and limited transport processes cause irreversibilities in the voltammetric behavior, complicating the analysis. No buffer solution without specific anion adsorption could be found in this $\mathrm{pH}$ range. Because anion adsorption could complicate the interpretation of T-jump measurements, experiments were limited to $\mathrm{pH}$ values above 11 .

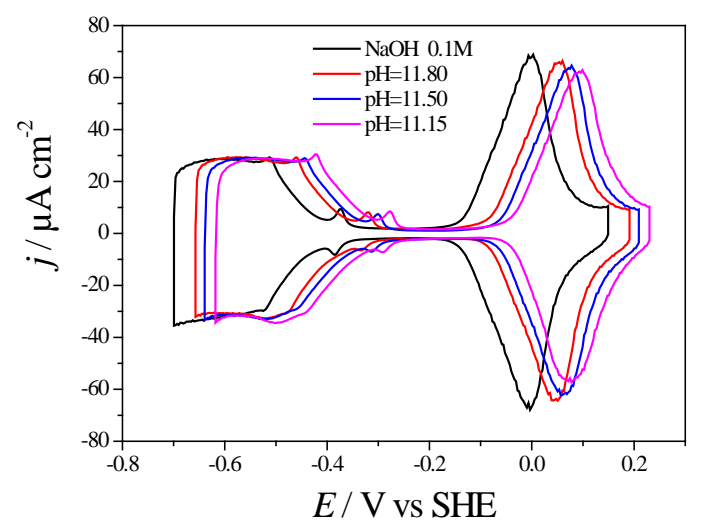

Figure 1: Voltammograms of a $\mathrm{Pt}(111)$ electrode in contact with $0.1 \mathrm{M} \mathrm{NaOH}+x \mathrm{M}$ $\mathrm{HClO}_{4}$ solutions at different $\mathrm{pH}$ values, as indicated in the figure. 
The corresponding laser induced potential transients recorded for $\mathrm{Pt}(111)$ in those solutions are shown in figure 2. Such potential transients are negative in all cases at potentials corresponding to the hydrogen adsorption-desorption zone. We have previously interpreted the sign of the T-jump induced potential transients as an indication of the orientation of water molecules at the interphase $[8,27,29]$. The negative sign suggests that water dipoles are oriented with the hydrogen end towards the surface. Such orientations gives a positive contribution to the potential drop between electrode and solution. The increase of the temperature decreases the positive dipolar contribution, resulting in a negative potential transient. This suggests that the pzfc is located above the hydrogen adsorption region. It is also worth recalling that, in acid solutions, the T-jump potential transients in the hydrogen region show a nonmonotonous behavior that has been interpreted as a result of hydrogen coverage changes taking place in the time scale of the measurement [27,29].

Increase of the $\mathrm{pH}$ decreases such contribution to an extent that was nearly negligible at $\mathrm{pH}$ above 3. Impedance measurements confirm that the rate of $\mathrm{H}$ adsorption is much slower in alkaline than in acid solution [39]. Hence, it is expected that hydrogen adsorption does not have enough time to respond in the time scale of the laser transient experiment in alkaline solutions, and, therefore, in this case, the response recorded in the hydrogen region would correspond mainly to the reorientation of water molecules. When the electrode potential is increased, the magnitude of the negative laser transient decreases, while it remains negative in the whole double layer potential range. It is only at the onset of the hydroxyl adsorption region that the potential transients become positive.

In acid or neutral media, the inversion of the sign of the transients takes place in the double layer or in the hydrogen adsorption region $[9,27,29]$. In those cases, the inversion potential, i.e. the potential value at which the laser transient changes the sign, has been taken as a reference value for the location of the potential of maximum entropy (pme). This means that the electric field is weak and the interfacial water structure is quite labile, showing maximum entropy. In those cases, the pme does not change significantly with $\mathrm{pH}$, remaining constant in the SHE scale at ca. $300 \mathrm{mV}$ vs SHE $[8,27]$. This result is consistent with the CO displacement measurements that show an invariability of extrapolated values of pzfc at different pHs also around the same potential value $[14,15]$. The reduction of peroxodisulfate shows consistent results with 
this interpretation [22]. The coincidence between those measurements supports the hypothesis that the response to the T-jump is mainly due to the reorientation of water and that the latter takes place around the pzfc.

The results shown in figure $2 \mathrm{~A}$, however, clearly demonstrate that the constancy in the potential where the inversion of the T-jump induced transient takes place just mentioned above is no longer held in the most alkaline solution. The laser induced temperature jump technique shows a bipolar transient at $-75 \mathrm{mV}$ vs SHE (690 mV vs RHE) at pH 13 $(0.1 \mathrm{M} \mathrm{NaOH})$ while the transient is definitely positive at $-50 \mathrm{mV}$ vs SHE, i.e., at a potential much lower than the value of the pzfc extrapolated from measurements in acid solutions, that is around $300 \mathrm{mV}$ vs SHE. Figure 2 also shows that the potential for the inversion of the signal does not remain constant but shifts with the onset of hydroxide adsorption to more positive potentials as the $\mathrm{pH}$ decreases. Inversion potentials from figure 2 are $-75 \mathrm{mV},-8 \mathrm{mV}, 60 \mathrm{mV}$ and above $131 \mathrm{mV}$ vs SHE for $\mathrm{pHs} 13,11.8,11.5$ and 11.15, respectively.
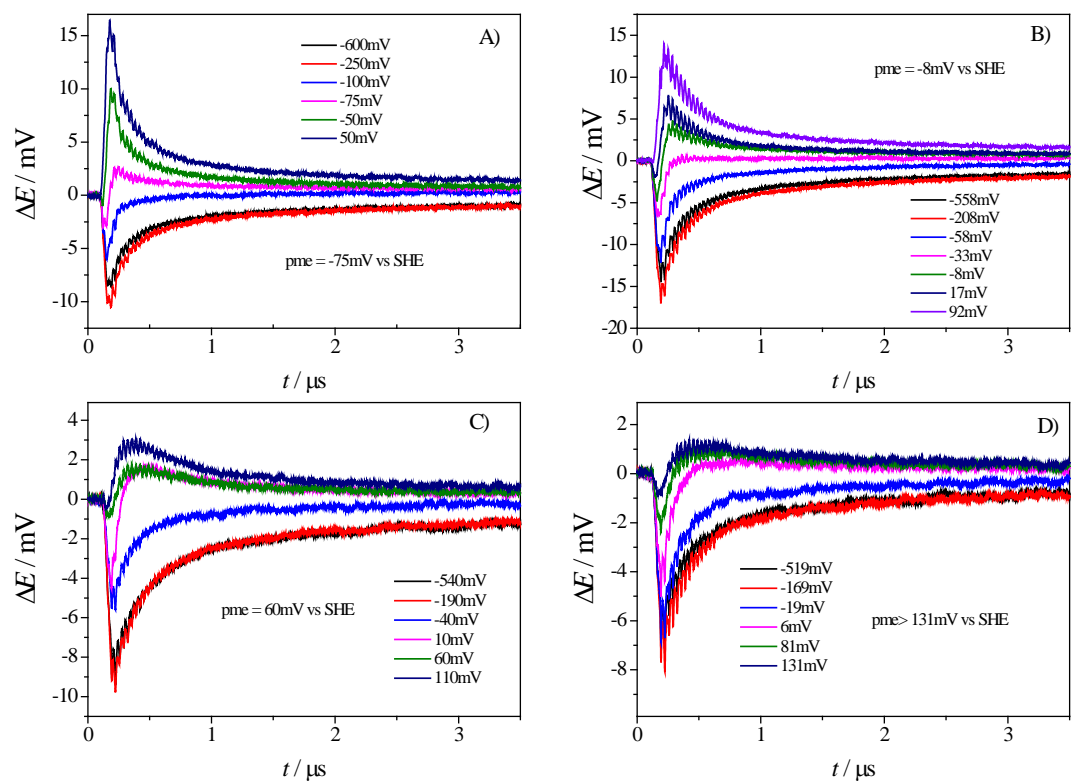

Figure 2. Laser-induced potential transients recorded for $\operatorname{Pt}(111)$ in $0.1 \mathrm{M} \mathrm{NaOH}+\mathrm{x} \mathrm{M}$ $\mathrm{HClO}_{4}$ solutions of different $\mathrm{pH}$ : a) $0.1 \mathrm{M} \mathrm{NaOH}$, b) $\mathrm{pH}=11.80$, c) $\mathrm{pH}=11.50$, d) $\mathrm{pH}=11.15$. Energy beam: 2-2.5mJ. All given potentials are in the SHE scale. 
Another point that deserves some comment is the observation of non-monotonous bipolar transients in the potential region where the laser response inversion takes place. As mentioned above, non-monotonous laser induced potential transients observed before were interpreted as the result of two overlapping processes with different rates $[27,29]$. Hence, while the pme should correspond to a nearly zero potential transient, bipolar transients like the ones in figure 2 can be the result of different phenomena with opposing sign and different time constant. At this point, it is important to recall that the potential shift with the laser perturbation contains different contributions in addition to the response of water dipoles [40,41]. First, the thermodiffusion potential appears as a consequence of the difference of temperatures between working and reference electrode, and second, the effect of the temperature on the surface potential of the metal electrode $(\Delta \chi)$ may also contribute to the potential transient. Although, in general, the contribution from water dipoles is the main one, close to the pme the dipolar contribution may become smaller and the other terms may become relatively more significant. In addition, it is also possible that close to the pzfc the reorientation of water molecules becomes slower, showing a bipolar profile. Still, it is clear that an inversion of the laser transient takes place at the onset of hydroxyl adsorption. While such bipolar shape produces some uncertainty in the determination of the pme, it is still easy to unambiguously locate the potential range where the transient change from clearly negative to clearly positive.

Another important observation from figure 2 is that the positive response from the laser transient at potentials above the inversion point tends to be slower, as the hydroxide concentration is decreased. This is reflected in a strong bipolar, non-monotonous character in the potential transient recorded at positive potentials in the lowest $\mathrm{pH}$ value studied here. This characteristic shape can be rationalized as the overlap of two signals with different time constant. We have previously shown that the slow response can be mathematically modelled as the convolution of the temperature change with an exponential decay, resulting in the following expression [8]:

$\square \Delta \square+\measuredangle 2 \square[\exp -\square \square \square \Delta \square \square \square \square$

$\triangle 1$ and $\bar{~} 2$ are the proportionality constants for the fast and slow responses, respectively is the time constant for the slow contribution. Considering that the fast response can also have a non-zero time constant and that the amplifier and the oscilloscope used for 
measuring the signal also have a finite time response, the previous equation can be improved as:

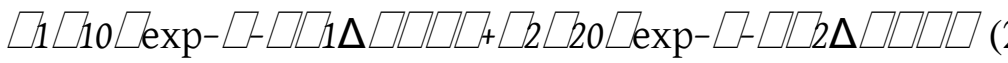

$\triangle 1$ and $[$ are the time constants for the fast and the slow response contributions, respectively. The fit of this equation to the experimental data can be done in the Laplace space:

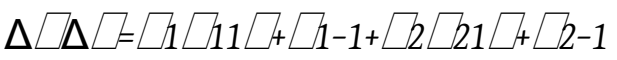

As an example, the result of the fit to the transients measured at the two highest potentials in figure $2 \mathrm{D}(\mathrm{pH}=11.15)$ is show in figure 3 . While the proportionality $\triangle 1$ and $Z_{2}$ are subject to large uncertainty because the actual temperature change cannot be measured, the value of time constants is rather insensitive to this uncertainty. Accordingly, time constants for the slow response are in the order of 0.3-0.4 $\mu \mathrm{s}$, while the time constant for the fast response is much lower, in the order of $20 \mathrm{~ns}$, which can be due to the response of the measuring amplifier.

The observation of these relatively large time constants rises some questions about the nature of such slow response. One possibility to explain the slow transient would be that the response of the water network to the temperature change is slow in mildly alkaline solutions but somehow catalyzed in strongly alkaline solution. A similar "acid" catalysis was proposed to explain the faster response in $0.1 \mathrm{M} \mathrm{HClO}_{4}$ in comparison with the solution with $\mathrm{pH}=3$ for stepped surfaces vicinal to $\operatorname{Pt}(111)$ [26]. This would mean that a large concentration of hydronium or hydroxyl species would facilitate the response of the water network to the thermal perturbation by altering the intermolecular hydrogen bond interaction. The results with stepped surfaces described next will shed some additional light on this issue.

Another possible explanation would be that the slow response is due to hydroxyl adsorption. Under this hypothesis, during the potential transient $\mathrm{OH}$ coverage would change in response to the potential shift. This change of coverage would release or withdraw electrons from the metal surface, affecting the shape of the transient. This would be analogous to the response obtained for $\mathrm{H}$ adsorption in acid media [27,29]. In alkaline media, it is reasonable to assume that $\mathrm{OH}$ adsorption will be much faster. In fact, impedance measurements revealed a significantly fast adsorption process in the hydroxyl region [42]. Then, as the $\mathrm{pH}$ is increased, the rate of $\mathrm{OH}$ adsorption would increase, in accordance with the experimental response in figure 2. Under this 
hypothesis, the positive transient at $\mathrm{pH} 13$ in the hydroxyl region would be dominated by an extremely fast $\mathrm{OH}$ adsorption process and, therefore, would not truly reflect the orientation of water. Instead, it would reflect a positive thermal coefficient for the adsorption process and a very large rate constant. However, additional experimental observations that do not fit with the previous explanation are obtained when the laser induced transients are measured at potentials above the $\mathrm{OH}$ adsorption region, as shown in figure 4. In this region, in which the $\mathrm{OH}$ adlayer attains full coverage, changes of coverage resulting from the potential shift as response to the T-jump should be very small, and, therefore, should have little influence on the potential transient. However, at those potential values, the laser induced transients still show a small positive response.

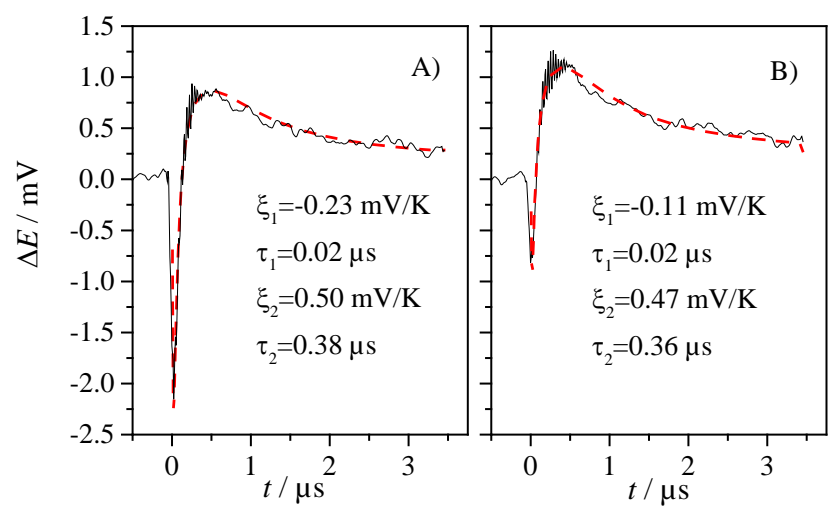

Figure 3: Fit of equation (2) to the transients measured at A) $81 \mathrm{mV}$ and B) $131 \mathrm{mV}$ at $\mathrm{pH} 11.15$ (figure 2D). The solid line is the experimental data and the dashed line the result of the fit. The parameters of the fit are shown in the figure.
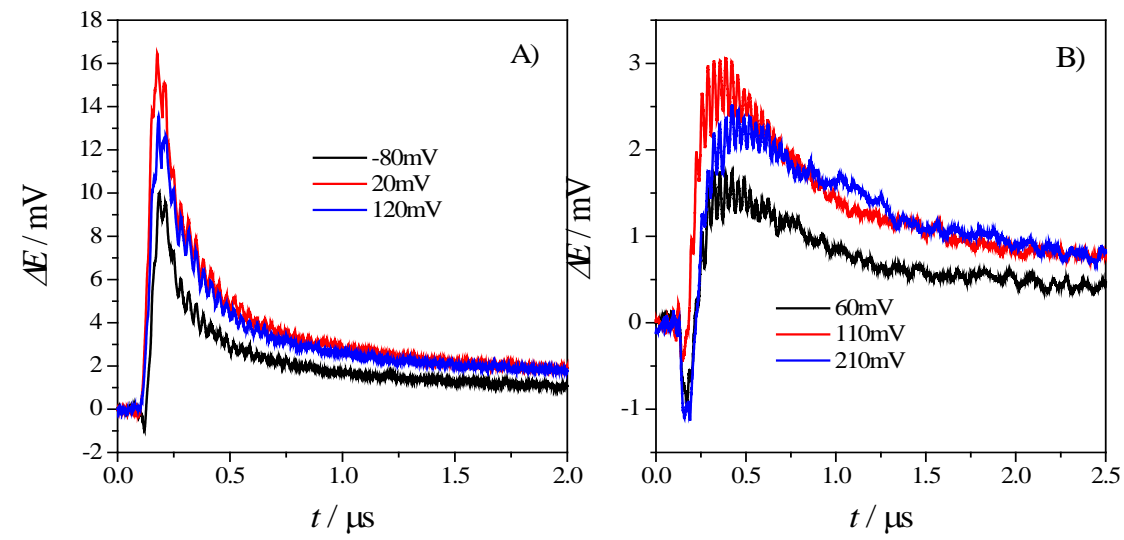

Figure 4. Laser-induced potential transients recorded for $\mathrm{Pt}(111)$ in $0.1 \mathrm{M} \mathrm{NaOH}+\mathrm{xM}$ $\mathrm{HClO}_{4}$ solutions of different $\mathrm{pH}$ at higher potentials: a) $\left.0.1 \mathrm{M} \mathrm{NaOH}, \mathrm{b}\right) \mathrm{pH}=11.50$. Energy beam: 2-2.5mJ. All given potentials are in the SHE scale. 


\section{Platinum stepped surfaces}

The laser induced T-jump study was extended to single crystal stepped surfaces containing (111) terraces. The use of stepped surfaces is key to understand the role of defects on the electronic properties of the surface, approaching more real systems such as polycrystalline electrodes or nanoparticles catalysts. The stepped surfaces employed here are those with the nominal structure $\operatorname{Pt}(S)[(n-1)(111) x(110)]$, i.e., (111) terraces $(n$ 1) atoms wide, separated by monoatomic steps of (110) symmetry. The Miller indices of these surfaces follow the common expression $\operatorname{Pt}(n, n, n-2)$ where $n$ represents the number of rows in the (111) terrace.

Figure 5 shows the cyclic voltammograms for $\operatorname{Pt}(553), \operatorname{Pt}(775), \operatorname{Pt}(554)$ and $\operatorname{Pt}(10,10,9)$ electrodes in $0.1 \mathrm{M} \mathrm{NaOH}$. The voltammograms obtained with these surfaces contain the main features described above for the Pt(111) electrode corresponding to hydrogen adsorption on the terrace below $0.35 \mathrm{~V}$ and hydroxyl adsorption above $0.65 \mathrm{~V}$. In addition, a reversible peak around $0.27 \mathrm{~V}$ appears in the hydrogen UPD region, and it is ascribed to the hydrogen adsorption-desorption on the (110) step sites [38]. The height of this peak grows as the step density increases, as expected, while the charge involved in the hydrogen and hydroxide adsorption region decreases.

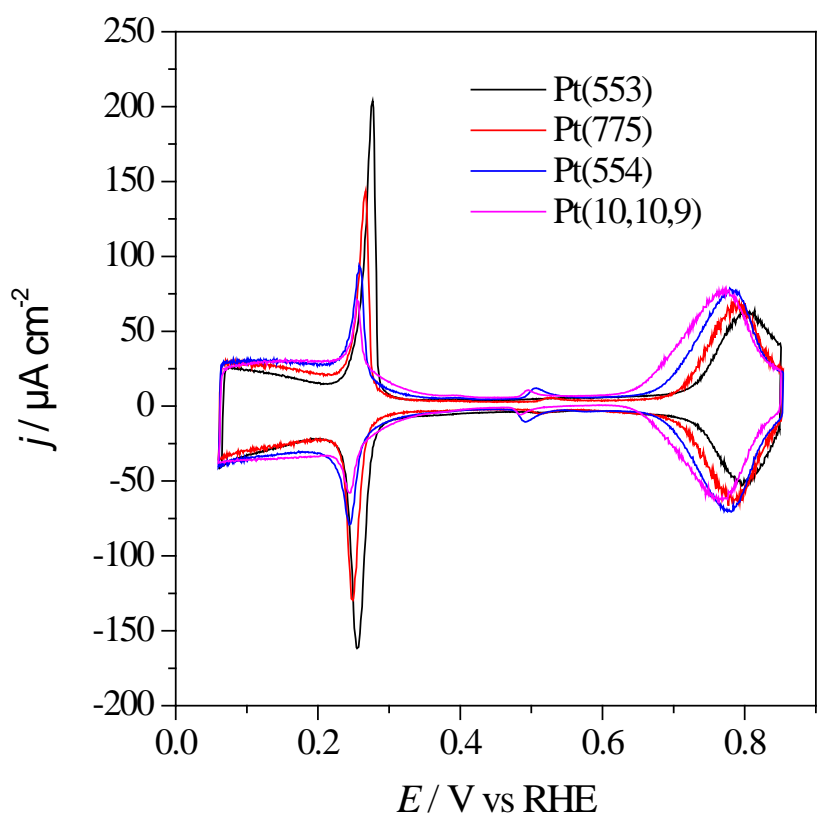


Figure 5. Cyclic voltammograms for $\operatorname{Pt}(\mathrm{S})[(\mathrm{n}-1)(111) \mathrm{x}(110)]$ stepped surfaces in $0.1 \mathrm{M}$ $\mathrm{NaOH}$. Scan rate: $50 \mathrm{mV} / \mathrm{s}$.

Laser induced potential transients of the different stepped surfaces were recorded following the same methodology described above. Laser transients are shown in figure 6. Potential transients are negative at the lowest potential limit, suggesting that water is oriented with an average dipole moment positive towards the surface. As the potential is increased, the magnitude of the transient decreases, inverting its sign around $0.25 \mathrm{~V}$. The potential where the inversion takes place coincides with the peak assigned to hydrogen adsorption on the step and therefore this phenomenon can be reasonably related to the presence of the steps. The inversion potential is nearly independent of the step density. With a further increase of the potential, the transients become negative with a second inversion from negative to positive values taking place in the double layer region. Such variation of the sign of the potential transients implies the existence of two relative maxima in the entropy of formation of the double layer (i.e., two values of the pme). This behavior was already reported for similar stepped surfaces in acid solution and has been interpreted in terms of the existence of local charge densities different for terrace and step sites [26,28]. This implies the existence of two values where the local free charge becomes zero, one related to the change of sign of the charge on steps ( $\mathrm{pzfc}_{\text {steps }}$ ) and the second one related to terrace sites but still affected by the presence of steps. It is worth noting that the inversion of the T-jump induced transients cannot be ascribed to the local reorganization of the water structure on steps or terraces, since the transients reflect the net response to the temperature change from the whole water adlayer. The existence of strong collaborative effects in the organization of the water network due to the strong hydrogen bonding in the adlayer was proposed to explain the sensitivity of the laser transients to local changes of the charge $[26,28]$. Under this interpretation, the change of the local electric field (step or terraces) would induce a disorder in the nearby water molecules that triggers a reorientation of the whole interfacial water network. In acid media, the consistency of this second pme with a pzfc related to the presence of steps and determined with other reaction probes, namely, $\mathrm{N}_{2} \mathrm{O}$ and peroxodisulfate reduction, supports this hypothesis $[10,18]$. 


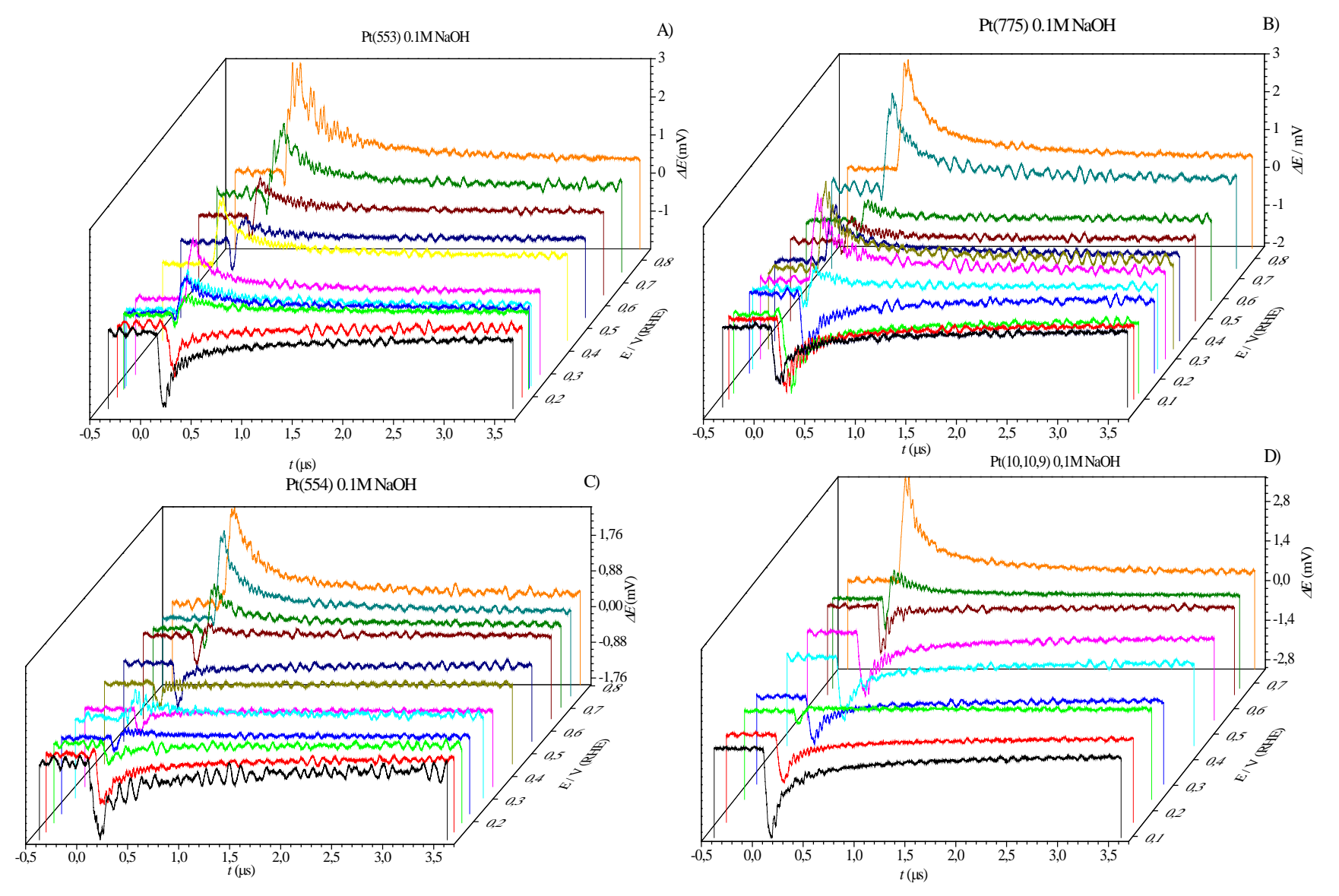

Figure 6. Laser transients for $\mathrm{Pt}(553), \operatorname{Pt}(775), \operatorname{Pt}(554)$ and $\mathrm{Pt}(10,10,9)$ in $0.1 \mathrm{M} \mathrm{NaOH}$.

Figure 7 compares the potential for the inversion of the transients associated to terrace and steps sites as a function of the step density, in acid and in alkaline solution. The pme value close to the local pzfc of steps remains nearly constant with the step density in both acid and alkaline solutions. There is a difference of $150 \mathrm{mV}$ between the values measured in the solution of $\mathrm{pH} 1$ and those measured at $\mathrm{pH} 13$ (Figure 7A). This shift corresponds to a slope of $12 \mathrm{mV} / \mathrm{pH}$ unit in the RHE scale (or $47 \mathrm{mV} / \mathrm{pH}$ unit in the SHE scale). This slope is similar to that determined between $\mathrm{pH} 1$ and 3 (ca $15 \mathrm{mV} / \mathrm{pH}$ unit), suggesting that the shift of the local pzfc of steps with $\mathrm{pH}$ is nearly linear and slightly smaller than the $59 \mathrm{mV} / \mathrm{pH}$ unit predicted from a purely Nernstian behavior. This deviation from the expected Nernstian behavior has been rationalized from the effect of cations [43], which will indeed affect that water network.

On the other hand, the dependence of the pme of the terraces with the step density in alkaline solutions is different from that observed in acid solutions. In acid solution, the 
pme of the terrace increases with the step density. Similar trend was also observed with the reduction of peroxodisulfate [10]. The disruption of the long range order in the water network induced by the introduction of steps was suggested to explain this shift of the pme to higher potential with the shortening of the terrace. It has been reported for gold $[25,44]$ and mercury $[40,45,46]$ electrodes that the pme is located at potential values lower than the pzfc. This is due to the existence of specific interactions between the oxygen atom in the water molecules and the surface. Then, it is necessary to apply negative charges to break this specific interaction to reach the potential of maximum disorientation. It is reasonable to accept that the existence of lateral interactions through strong hydrogen bonding in the water network will to tend to stabilize the configuration with the oxygen towards the metal. Then, the stronger the lateral interactions in the water network, the larger the difference between the pme and the pzfc. Under this hypothesis, introduction of steps would have the effect of destabilizing the water network shifting the pme towards the pzfc, i.e., towards higher values. Similar effect was described with different adatoms of $s^{2} \mathrm{p}^{\mathrm{n}}$ semimetals deposited at low coverage on $\operatorname{Pt}(111)[30]$.

In alkaline solution, the pme of the terraces decreases as the step density is increased. One possibility to justify this difference could be that the presence of hydroxyl anions in the proximity of the surface significantly affects the network of hydrogen bonds, changing the preferential orientation of water. If the water network is stabilized through lateral interactions with the hydrogen towards the metal, the disruption of the network due to the presence of the step would shift the pme toward lower values, as observed in figure 7. Another plausible explanation for this discrepancy would be that, in alkaline solutions, it is likely that hydroxyl adsorption on step sites is more favored. Importantly, anion adsorption on step sites in acid solution is unlikely to take place, since the characteristic voltammetric features related to steps are not affected by the anion in solution [6]. In alkaline solution, hydroxide adsorption on the steps would induce positive image charge on the adjacent terrace sites, which would induce net orientation of the water molecules with the oxygen towards the metal. Under this scenario, the reorientation of the water molecules on the terrace would take place at increasingly more negative potentials as the step density increases. A similar interplay of induced charges was proposed in [47], although detailed computational studies in this regard are still lacking. 

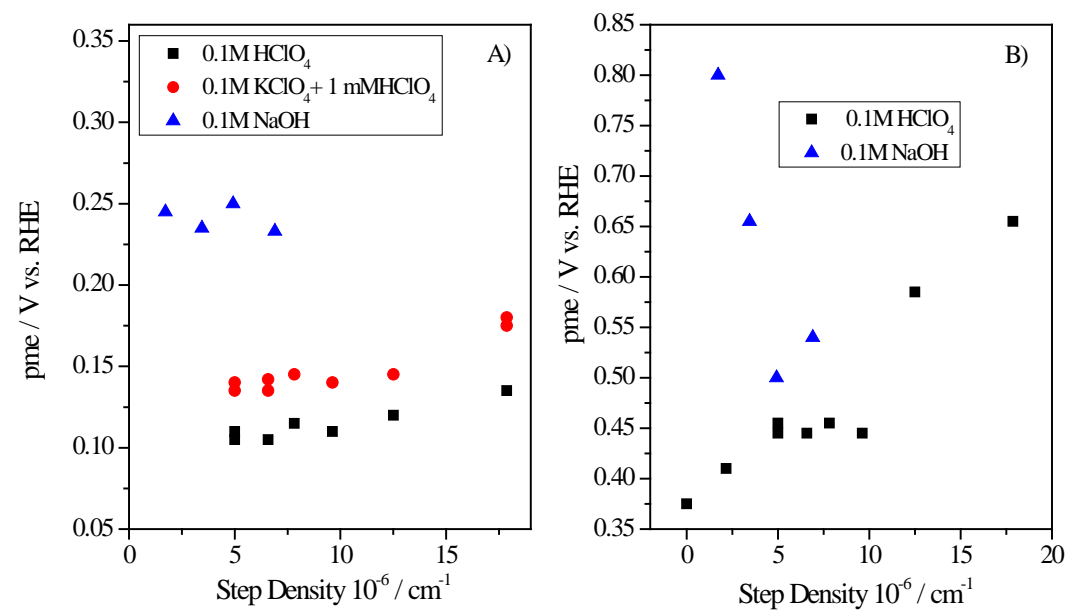

Figure 7. Values of pme associated to A) the step and B) terraces sites for $\operatorname{Pt}(\mathrm{S})[(\mathrm{n}$ 1)(111)x(110)] surfaces in $0.1 \mathrm{M} \mathrm{HClO}_{4}$ (squares), $0.1 \mathrm{M} \mathrm{KClO}_{4}+1 \mathrm{mM} \mathrm{HClO}_{4}$ (circles) and $0.1 \mathrm{M} \mathrm{NaOH}$ (triangles). The obtained values for $\mathrm{pH} 1$ and $\mathrm{pH} 3$ are taken from reference [26]

To further complete the study of the effect of the surface orientation on the pme, the laser induced transients were also recorded for the other two basal planes, $\operatorname{Pt}(100)$ and $\mathrm{Pt}(110)$, in $0.1 \mathrm{M} \mathrm{NaOH}$. The corresponding blank cyclic voltammograms for these surfaces are shown in Figure 8. The characteristic voltammetric profiles agree with the results previously published in the literature showing high cleanliness level and surface ordering [13,48]. For Pt(110), a single peak around $0.24 \mathrm{~V}$ vs RHE overlaps with a second broader peak or shoulder. A similar voltammogram is recorded in sulfuric acid media and has been explained considering that both peaks contain contributions for hydrogen and hydroxyl adsorption desorption [49-51]. The separation into peak and shoulder may reflect the existence of patches on the surface with different degrees of (1x2) reconstruction [50,51]. For Pt(100), a multiplicity of peaks are observed, which can be attributed to hydrogen and hydroxyl adsorption on both 2D ordered terraces and monodimiensional (100) sites [13,52]. To explain the presence of the latter, we need to recall that the pretreatment of the electrode at high temperatures induces a thermal surface reconstruction [53], which is lifted when the electrode is brought into contact 
with the solution under potential control conditions [54]. The excess atoms on the surface after lifting the reconstructions form mesa-like structures increasing the density of monodimensional (100) sites [55-57].

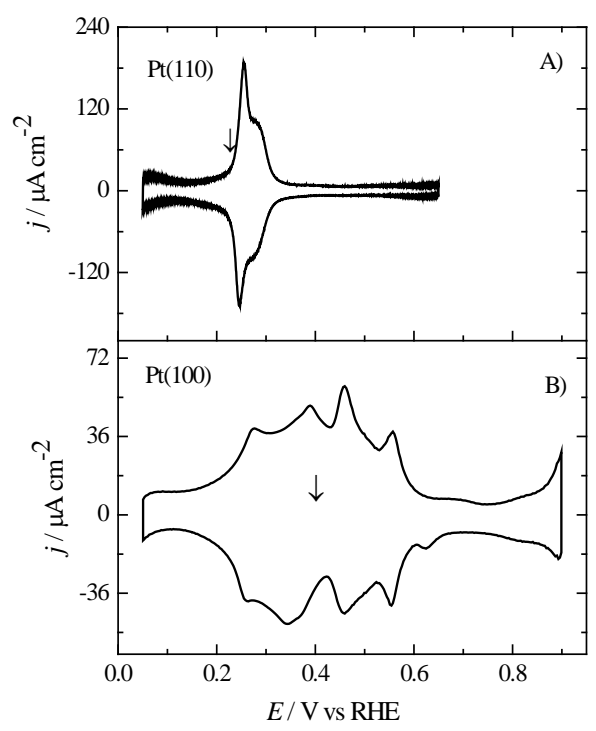

Figure 8. Voltammograms corresponding to A) Pt (110) and B) Pt(100) in $0.1 \mathrm{M} \mathrm{NaOH}$. The arrows mark the position of the inversion of the sign of the laser induced potential transients as shown in figure 9 . Scan rate: $50 \mathrm{mV} / \mathrm{s}$.

For these two surfaces, laser induced potential transients are negative at low enough potentials, i.e., the temperature coefficient of the double-layer is negative (Figure 9). At these potentials, the net orientation of the interfacial water molecules is with the hydrogens pointing towards the surface. Similarly to the $\operatorname{Pt}(111)$ orientation, the sign of the transients change from negative to positive when the potential of the experiment is increased above a given value. The potentials for the inversion of the transients are 0.68 , 0.40 and $0.23 \mathrm{~V}$ for $\mathrm{Pt}(111), \operatorname{Pt}(100)$ and $\mathrm{Pt}(110)$, respectively. This trend is the same as in acid solutions [27] and also agrees with the expectation based on the work functions values [58]. It is worth noting that the potential for the inversion of the transients found here for $\mathrm{Pt}(100)$ and $\mathrm{Pt}(110)$ in alkaline solutions are very close (in the RHE scale) to the values previously reported in $0.1 \mathrm{M} \mathrm{HClO}_{4}$ solution, 0.35 and $0.15 \mathrm{~V}$, respectively [27]. Considering the whole range of $\mathrm{pH}$ spanned between both studies, from 1 to 13, this shift would correspond to a slope of 7 and $4 \mathrm{mV} / \mathrm{pH}$ unit, respectively, in the RHE 
scale (-52 and $-55 \mathrm{mV} / \mathrm{pH}$ in the SHE scale). However, the shift of the pme with the $\mathrm{pH}$ must be non-linear since much higher slope was detected in acidic solutions (a shift of 10 and $26 \mathrm{mV} / \mathrm{pH}$ in the RHE scale was detected for $\mathrm{Pt}(110)$ and $\operatorname{Pt}(100)$, respectively, between $\mathrm{pH}=1$ and 4). It should also be noted that the pme for $\operatorname{Pt}(110)$ is located at the onset of hydrogen desorption peak (close to the completion of the hydrogen adlayer). This is different in acid solution where the pme is slightly higher than the potential of the main peak [27]. Also, the pme of $\mathrm{Pt}(100)$ is slightly lower than the position of the main peak unlike in acid solution.
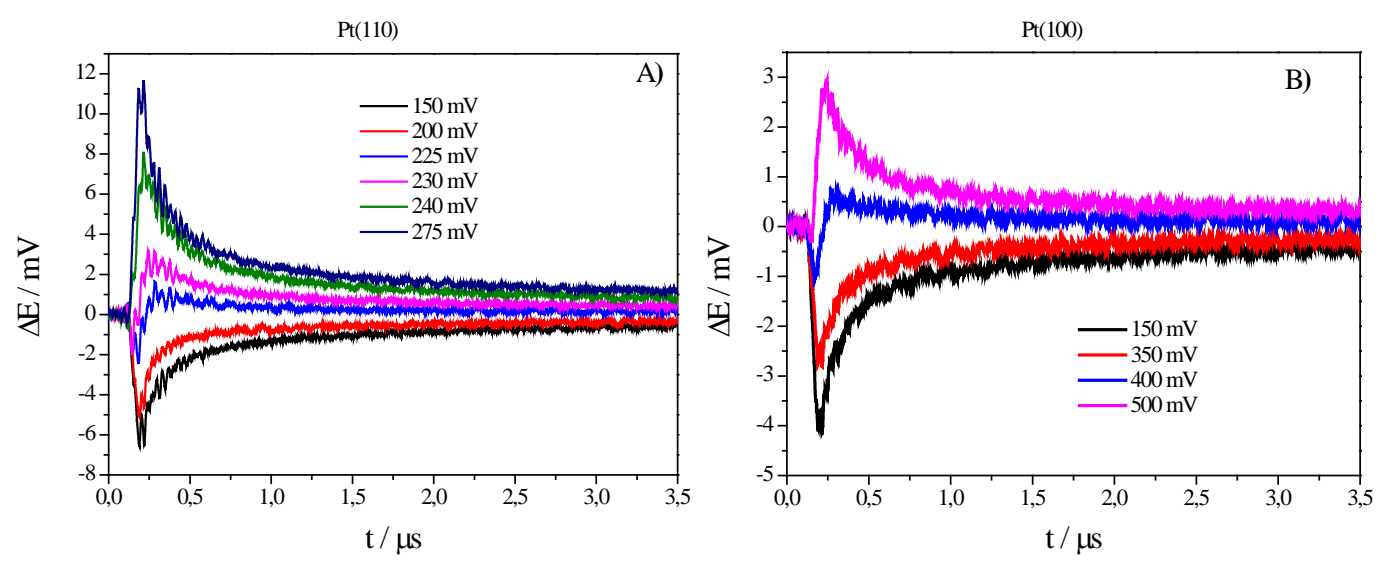

Figure 9: laser-induced potential transients at different potentials (vs RHE), as indicated on the figure on $\mathrm{A}) \mathrm{Pt}(110)$ and $\mathrm{B}) \mathrm{Pt}(100)$ in $0.1 \mathrm{M} \mathrm{NaOH}$. Energy beam: $1 \mathrm{~mJ}$

Similarly to the result described above for Pt(111), bipolar transients (figure 10) signaling the existence of a slow response are also obtained for $\operatorname{Pt}(100)$ and $\operatorname{Pt}(110)$ when the $\mathrm{pH}$ is decreased below 12. More work is in progress to better understand the nature of such bipolar transients.
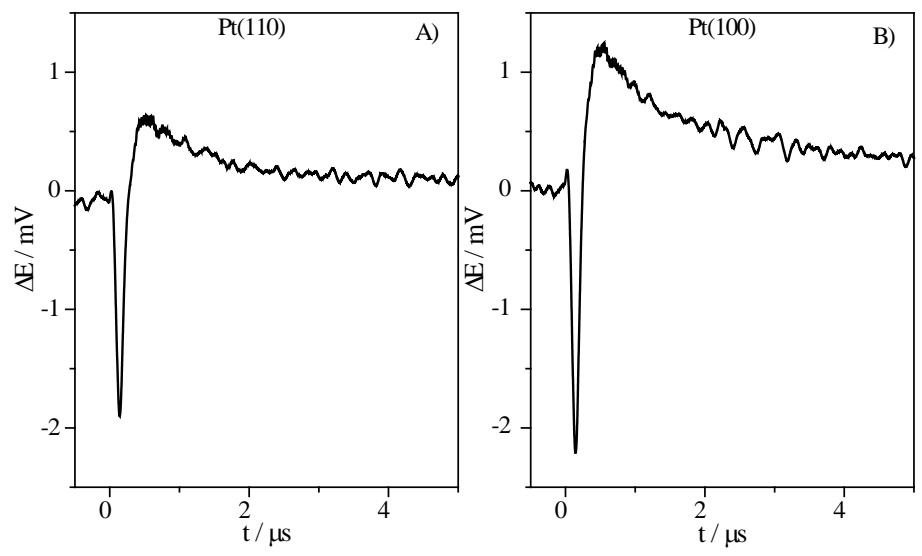
Figure 10: laser-induced potential transients at $\mathrm{pH}=11.90$ on $\mathrm{A}) \operatorname{Pt}(110)$ at $0.52 \mathrm{~V}$ RHE and B) Pt(100) at 0.36 V RHE. Energy beam: 2-2.5mJ.

\section{Conclusions}

The interphase of platinum single crystal surfaces in contact with alkaline aqueous solutions has been investigated using the laser induced temperature jump method. The sign of the coulostatic potential transients measured as response to the temperature increase has been analyzed to extract information about the water orientation and its relation with the charge separation at the interphase. In all cases, the laser induced potential transients are negative at low potentials close to the onset of hydrogen evolution. When the potential is increased, the magnitude of the transient decreases, in agreement with a decrease in the magnitude of the electric field, until an inversion in the sign of the transient takes place. For the platinum basal planes, the inversion follows the order $\operatorname{Pt}(110)<\operatorname{Pt}(100)<\operatorname{Pt}(111)$. This trend agrees with results obtained in acid solutions and work function expectations. After this inversion, the transients remain positive even after the completion of the adsorption processes.

For the three basal planes, the potential where transients change sign is higher than the value detected in acid solution in the RHE scale. This has important consequences to understand the effect of charge on water polarization and its influence on reactivity. For instance, it has been proposed that the main reason for the significant difference in the rate of hydrogen evolution between acid and alkaline solution stems from the more rigid polarization of water as a consequence of a stronger electric field at the higher $\mathrm{pH}$ $[59,60]$.

The laser induced potential transients were also used to investigate stepped surfaces vicinal to the (111) surface. In this case, two inversion potentials can be identified with the existence of two local pzfc values, associated to terrace and step sites. The local pzfc associated to step sites remains independence of the step density. However, the local pzfc of the terrace decreases when the step density is increased. This is in clear contrast with the result obtained in acid solution. Tentative explanations based on the possible effect of hydroxyl anions on the water structure or, alternatively, on the induction of charges on the terrace by the anion adsorbed on step sites have been discussed. 
These results will serve to better understand the effect of $\mathrm{pH}$ on the interfacial properties of platinum and its influence on electrochemical reactivity.

\section{Acknowledgements}

This work has been financially supported by the MINECO (Spain) project No. CTQ2016-76221-P.

\section{References.}

[1] J.O. Bockris, M.A.V. Devanathan, K. Muller, On the structure of charged interfaces, Proc. R. Soc. London. Ser. A. Math. Phys. Sci. 274 (1963) 55-79. https://doi.org/10.1098/rspa.1963.0114.

[2] T. Roman, A. Groß, Structure of water layers on hydrogen-covered Pt electrodes, Catal. Today. 202 (2013) 183-190. https://doi.org/10.1016/j.cattod.2012.06.001.

[3] A.N. Frumkin, O.A. Petrii, B.B. Damaskin, Potentials of Zero Charge, in: Compr. Treatise Electrochem., 1980. https://doi.org/10.1007/978-1-4615-66847_5.

[4] S. Trasatti, E. Lust, The Potential of Zero Charge, in: Mod. Asp. Electrochem., 2005. https://doi.org/10.1007/0-306-46917-0_1.

[5] V. Climent, R. Gómez, J.M. Feliu, Effect of increasing amount of steps on the potential of zero total charge of Pt(111) electrodes, Electrochim. Acta. 45 (1999) 629-637. https://doi.org/10.1016/S0013-4686(99)00241-8.

[6] R. Gómez, V. Climent, J.M. Feliu, M.J. Weaver, Dependence of the Potential of Zero Charge of Stepped Platinum (111) Electrodes on the Oriented Step-Edge Density: Electrochemical Implications and Comparison with Work Function Behavior, J. Phys. Chem. B. 104 (2000) 597-605. https://doi.org/10.1021/jp992870c.

[7] N. Garcia Araez, V. Climent, E. Herrero, J. Feliu, J. Lipkowski, Thermodynamic approach to the double layer capacity of a $\mathrm{Pt}(111)$ electrode in perchloric acid solutions, Electrochim. Acta. 51 (2006) 3787-3793. https://doi.org/10.1016/j.electacta.2005.10.043. 
[8] P. Sebastián, R. Martínez-Hincapié, V. Climent, J.M. Feliu, Study of the Pt (111) | electrolyte interface in the region close to neutral $\mathrm{pH}$ solutions by the laser induced temperature jump technique, Electrochim. Acta. 228 (2017) 667-676. https://doi.org/10.1016/j.electacta.2017.01.089.

[9] R. Martínez-Hincapié, P. Sebastián-Pascual, V. Climent, J.M. Feliu, Investigating interfacial parameters with platinum single crystal electrodes, Russ. J. Electrochem. 53 (2017) 227-236. https://doi.org/10.1134/S1023193517030107.

[10] R. Martínez-Hincapié, V. Climent, J.M. Feliu, Investigation of the interfacial properties of platinum stepped surfaces using peroxodisulfate reduction as a local probe, Electrochim. Acta. 307 (2019) 553-563. https://doi.org/10.1016/j.electacta.2019.03.198.

[11] T. Pajkossy, D.M. Kolb, On the origin of the double layer capacitance maximum of Pt(1 1 1) single crystal electrodes, Electrochem. Commun. 5 (2003) 283-285. https://doi.org/10.1016/S1388-2481(03)00046-8.

[12] K. Ojha, N. Arulmozhi, D. Aranzales, M.T.M. Koper, Double Layer at the Pt(111)-Aqueous Electrolyte Interface: Potential of Zero Charge and Anomalous Gouy-Chapman Screening, Angew. Chemie Int. Ed. 59 (2020) 711-715. https://doi.org/10.1002/anie.201911929.

[13] R.M. Arán-Ais, M.C. Figueiredo, F.J. Vidal-Iglesias, V. Climent, E. Herrero, J.M. Feliu, On the behavior of the $\operatorname{Pt}\left(\begin{array}{lll}1 & 0 & 0\end{array}\right)$ and vicinal surfaces in alkaline media, Electrochim. Acta. 58 (2011) 184-192. https://doi.org/10.1016/j.electacta.2011.09.029.

[14] R. Martínez-Hincapié, P. Sebastián-Pascual, V. Climent, J.M. Feliu, Exploring the interfacial neutral $\mathrm{pH}$ region of $\mathrm{Pt}(111)$ electrodes, Electrochem. Commun. 58 (2015) 62-64. https://doi.org/10.1016/j.elecom.2015.06.005.

[15] R. Rizo, E. Sitta, E. Herrero, V. Climent, J.M. Feliu, Towards the understanding of the interfacial pH scale at Pt(111) electrodes, Electrochim. Acta. 162 (2015) 138-145. https://doi.org/10.1016/j.electacta.2015.01.069.

[16] V. Climent, N. García-Araez, E. Herrero, J. Feliu, Potential of zero total charge of platinum single crystals: A local approach to stepped surfaces vicinal to 
Pt(111), Russ. J. Electrochem. 42 (2006) 1145-1160.

https://doi.org/10.1134/S1023193506110012.

[17] G.A. Attard, A. Ahmadi, Anion—surface interactions Part 3. N2O reduction as a chemical probe of the local potential of zero total charge, J. Electroanal. Chem. 389 (1995) 175-190. https://doi.org/10.1016/0022-0728(95)03927-9.

[18] V. Climent, G.A. Attard, J.M. Feliu, Potential of zero charge of platinum stepped surfaces: a combined approach of CO charge displacement and N2O reduction, J. Electroanal. Chem. 532 (2002) 67-74. https://doi.org/10.1016/S00220728(02)00849-5.

[19] G.A. Attard, O. Hazzazi, P.B. Wells, V. Climent, E. Herrero, J.M. Feliu, On the global and local values of the potential of zero total charge at well-defined platinum surfaces: stepped and adatom modified surfaces, J. Electroanal. Chem. 568 (2004) 329-342. https://doi.org/10.1016/j.jelechem.2004.02.007.

[20] R. Martínez-Hincapié, V. Climent, J.M. Feliu, New probes to surface free charge at electrochemical interfaces with platinum electrodes, Curr. Opin. Electrochem. 14 (2019) 16-22. https://doi.org/10.1016/j.coelec.2018.09.012.

[21] V. Climent, M.D. Maciá, E. Herrero, J.M. Feliu, O. a. Petrii, Peroxodisulphate reduction as a novel probe for the study of platinum single crystal/solution interphases, J. Electroanal. Chem. 612 (2008) 269-276. https://doi.org/10.1016/j.jelechem.2007.10.009.

[22] R. Martínez-Hincapié, V. Climent, J.M. Feliu, Peroxodisulfate reduction as a probe to interfacial charge, Electrochem. Commun. 88 (2018) 43-46. https://doi.org/10.1016/j.elecom.2018.01.012.

[23] R. Martínez-Hincapié, V. Climent, J.M. Feliu, Peroxodisulfate reduction on platinum stepped surfaces vicinal to the (110) and (100) poles, J. Electroanal. Chem. 847 (2019) 113226. https://doi.org/10.1016/j.jelechem.2019.113226.

[24] V. Briega-Martos, E. Herrero, J.M. Feliu, Effect of $\mathrm{pH}$ and Water Structure on the Oxygen Reduction Reaction on platinum electrodes, Electrochim. Acta. 241 (2017) 497-509. https://doi.org/10.1016/j.electacta.2017.04.162.

[25] V. Climent, B.A. Coles, R.G. Compton, Laser-Induced Potential Transients on a $\mathrm{Au}(111)$ Single-Crystal Electrode. Determination of the Potential of Maximum 
Entropy of Double-Layer Formation, J. Phys. Chem. B. 106 (2002) 5258-5265. https://doi.org/10.1021/jp020054q.

[26] N. Garcia Araez, V. Climent, J. Feliu, Potential-dependent water orientation on Pt(111) stepped surfaces from laser-pulsed experiments, Electrochim. Acta. 54 (2009) 966-977. https://doi.org/10.1016/j.electacta.2008.08.016.

[27] N. Garcia-Araez, V. Climent, J. Feliu, Potential-Dependent Water Orientation on Pt(111), Pt(100), and Pt(110), As Inferred from Laser-Pulsed Experiments. Electrostatic and Chemical Effects, J. Phys. Chem. C. 113 (2009) 9290-9304. https://doi.org/10.1021/jp900792q.

[28] V. Climent, B.A. Coles, R.G. Compton, J.M. Feliu, Coulostatic potential transients induced by laser heating of platinum stepped electrodes: Influence of steps on the entropy of double layer formation, J. Electroanal. Chem. 561 (2004) 157-165. https://doi.org/10.1016/j.jelechem.2003.07.029.

[29] V. Climent, B. a. Coles, R.G. Compton, Coulostatic Potential Transients Induced by Laser Heating of a Pt(111) Single-Crystal Electrode in Aqueous Acid Solutions. Rate of Hydrogen Adsorption and Potential of Maximum Entropy, J. Phys. Chem. B. 106 (2002) 5988-5996. https://doi.org/10.1021/jp020785q.

[30] N. García-Aráez, V. Climent, J.M. Feliu, Evidence of water reorientation on model electrocatalytic surfaces from nanosecond-laser-pulsed experiments., J. Am. Chem. Soc. 130 (2008) 3824-33. https://doi.org/10.1021/ja0761481.

[31] J. Huang, A. Malek, J. Zhang, M.H. Eikerling, Non-monotonic Surface Charging Behavior of Platinum: A Paradigm Change, J. Phys. Chem. C. 120 (2016) 13587-13595. https://doi.org/10.1021/acs.jpcc.6b03930.

[32] J. Clavilier, D. Armand, S.G. Sun, M. Petit, Electrochemical adsorption behaviour of platinum stepped surfaces in sulphuric acid solutions, J. Electroanal. Chem. Interfacial Electrochem. 205 (1986) 267-277. https://doi.org/10.1016/0022-0728(86)90237-8.

[33] C. Korzeniewski, V. Climent, J. Feliu, Electrochemistry at Platinum Single Crystal Electrodes, in: A.J. Bard, C.G. Zoski (Eds.), Electroanal. Chem. A Ser. Adv., CRC Press, Boca Raton, 2012: pp. 75-170. https://doi.org/10.1201/b11480-3. 
[34] J. Clavilier, K.E. Actii, M. Petit, A. Rodes, M.A. Zamakhchari, Electrochemical monitoring of the thermal reordering of platinum single-crystal surfaces after metallographic polishing from the early stage to the equilibrium surfaces, $\mathrm{J}$. Electroanal. Chem. Interfacial Electrochem. 295 (1990) 333-356. https://doi.org/10.1016/0022-0728(90)85026-2.

[35] R.A. Goffe, A.C. Tseung, Internally charged palladium hydride reference electrode - Part 1: The effect of charging current density on long-term stability, Med. Biol. Eng. Comput. 16 (1978) 670-676. https://doi.org/10.1007/BF02442446.

[36] N.S. Marinković, N.M. Marković, R.R. Adžić, Hydrogen adsorption on singlecrystal platinum electrodes in alkaline solutions, J. Electroanal. Chem. 330 (1992) 433-452. https://doi.org/10.1016/0022-0728(92)80323-V.

[37] E. Morallón, J.L. Vázquez, A. Aldaz, Electrochemical behaviour of Pt(111) in alkaline media. Effect of specific adsorption of anions, J. Electroanal. Chem. 334 (1992) 323-338. https://doi.org/10.1016/0022-0728(92)80581-N.

[38] M.J.S. Farias, C. Busó-Rogero, R. Gisbert, E. Herrero, J.M. Feliu, Influence of the CO adsorption environment on its reactivity with (111) terrace sites in stepped Pt electrodes under alkaline media, J. Phys. Chem. C. 118 (2014) 19251934. https://doi.org/10.1021/jp408975t.

[39] K.J.P. Schouten, M.J.T.C. Van Der Niet, M.T.M. Koper, Impedance spectroscopy of $\mathrm{H}$ and $\mathrm{OH}$ adsorption on stepped single-crystal platinum electrodes in alkaline and acidic media, Phys. Chem. Chem. Phys. 12 (2010) 15217-15224. https://doi.org/10.1039/c0cp00104j.

[40] V.A. Benderskii, G.I. Velichko, Temperature jump in electric double-layer study, J. Electroanal. Chem. Interfacial Electrochem. 140 (1982) 1-22. https://doi.org/10.1016/0368-1874(82)85295-7.

[41] J.F. Smalley, C. V. Krishnan, M. Goldman, S.W. Feldberg, I. Ruzic, Laserinduced temperature-jump coulostatics for the investigation of heterogeneous rate processes. Theory and application, J. Electroanal. Chem. 248 (1988) 255-282. https://doi.org/10.1016/0022-0728(88)85090-3.

[42] E. Sibert, R. Faure, R. Durand, High frequency impedance measurements on Pt ( 
111 ) in sulphuric and perchloric acids, J. Electroanal. Chem. 515 (2001) 71-81.

[43] X. Chen, I.T. McCrum, K.A. Schwarz, M.J. Janik, M.T.M. Koper, Co-adsorption of Cations as the Cause of the Apparent pH Dependence of Hydrogen Adsorption on a Stepped Platinum Single-Crystal Electrode, Angew. Chemie Int. Ed. 56 (2017) 15025-15029. https://doi.org/10.1002/anie.201709455.

[44] A. Hamelin, L. Stoicoviciu, F. Silva, The temperature dependence of the doublelayer properties of gold faces in perchloric acid solutions. Part I. the (210) gold face, J. Electroanal. Chem. 229 (1987) 107-124. https://doi.org/10.1016/00220728(87)85134-3.

[45] J.A. Harrison, J.E.B. Randles, D.J. Schiffrin, The entropy of formation of the mercury-aqueous solution interface and the structure of the inner layer, J. Electroanal. Chem. Interfacial Electrochem. 48 (1973) 359-381. https://doi.org/10.1016/S0022-0728(73)80369-9.

[46] R. Guidelli, G. Aloisi, E. Leiva, W. Schmickler, Contribution to the entropy of formation of metal/solution interphases from metal electrons, J. Phys. Chem. 92 (1988) 6671-6675. https://doi.org/10.1021/j100334a036.

[47] G.A. Attard, A phenomenological theory of electrosorption, J. Electroanal. Chem. 819 (2018) 481-494. https://doi.org/10.1016/j.jelechem.2017.12.057.

[48] E. Herrero, Q.S. Chen, J. Hernández, S.G. Sun, J.M. Feliu, Effects of the surface mobility on the oxidation of adsorbed CO on platinum electrodes in alkaline media. the role of the adlayer and surface defects, Phys. Chem. Chem. Phys. 13 (2011) 16762-16771. https://doi.org/10.1039/c1cp21909j.

[49] R. Gómez, J. Clavilier, Electrochemical behaviour of platinum surfaces containing (110) sites and the problem of the third oxidation peak, J. Electroanal. Chem. 354 (1993) 189-208. https://doi.org/10.1016/0022-0728(93)80333-D.

[50] G.A. Attard, A. Brew, Cyclic voltammetry and oxygen reduction activity of the Pt\{1 110$\}-(1 \times 1)$ surface, J. Electroanal. Chem. 747 (2015) 123-129. https://doi.org/10.1016/j.jelechem.2015.04.017.

[51] G.A. Attard, K. Hunter, E. Wright, J. Sharman, R. Martínez-Hincapié, J.M. Feliu, The voltammetry of surfaces vicinal to $\operatorname{Pt}\{110\}$ : Structural complexity simplified by CO cooling, J. Electroanal. Chem. 793 (2017) 137-146. 
https://doi.org/10.1016/j.jelechem.2016.10.005.

[52] P. Rodríguez, G. García, E. Herrero, J.M. Feliu, M.T.M. Koper, Effect of the Surface Structure of $\mathrm{Pt}(100)$ and $\mathrm{Pt}(110)$ on the Oxidation of Carbon Monoxide in Alkaline Solution: An FTIR and Electrochemical Study, Electrocatalysis. 2 (2011) 242-253. https://doi.org/10.1007/s12678-011-0059-9.

[53] M.A. Van Hove, R.J. Koestner, P.C. Stair, J.P. Bibérian, L.L. Kesmodel, I. BartoŠ, G.A. Somorjai, The surface reconstructions of the (100) crystal faces of iridium, platinum and gold, Surf. Sci. 103 (1981) 189-217. https://doi.org/10.1016/0039-6028(81)90107-2.

[54] A. Al-Akl, G.A. Attard, R. Price, B. Timothy, Voltammetric and UHV characterisation of the $(1 \times 1)$ and reconstructed hex-R0.7 $7^{\circ}$ phases of $\operatorname{Pt}\{100\}$, J. Electroanal. Chem. 467 (1999) 60-66. https://doi.org/10.1016/S00220728(99)00038-8.

[55] A. V. Rudnev, T. Wandlowski, An influence of pretreatment conditions on surface structure and reactivity of $\mathrm{Pt}(100)$ towards CO oxidation reaction, Russ. J. Electrochem. 48 (2012) 259-270. https://doi.org/10.1134/S1023193512030123.

[56] L.A. Kibler, A. Cuesta, M. Kleinert, D.M. Kolb, In-situ STM characterization of the surface morphology of platinum single crystal electrodes as a function of their preparation, J. Electroanal. Chem. 484 (2000) 73-82. https://doi.org/10.1016/S0022-0728(00)00065-6.

[57] J. Clavilier, J.M. Orts, J.M. Feliu, Etude de l'effet de prétraitements sur la topographie des surfaces orientées de platine par deux méthodes indépendantes : voltammétrie et STM, Le J. Phys. IV. 04 (1994) C1-303-C1-308. https://doi.org/10.1051/jp4:1994122.

[58] G.N. Derry, M.E. Kern, E.H. Worth, Recommended values of clean metal surface work functions, J. Vac. Sci. Technol. A Vacuum, Surfaces, Film. 33 (2015) 060801. https://doi.org/10.1116/1.4934685.

[59] I. Ledezma-Yanez, W.D.Z. Wallace, P. Sebastián-Pascual, V. Climent, J.M. Feliu, M.T.M. Koper, Interfacial water reorganization as a pH-dependent descriptor of the hydrogen evolution rate on platinum electrodes, Nat. Energy. 2 
(2017) 1-7. https://doi.org/10.1038/nenergy.2017.31.

[60] F.J. Sarabia, P. Sebastián-Pascual, M.T.M. Koper, V. Climent, J.M. Feliu, Effect of the Interfacial Water Structure on the Hydrogen Evolution Reaction on $\operatorname{Pt}(111)$ Modified with Different Nickel Hydroxide Coverages in Alkaline Media, ACS Appl. Mater. Interfaces. 11 (2019) 613-623. https://doi.org/10.1021/acsami.8b15003. 